\title{
Cooperative Automation Supporting Pilot-Dispatch Negotiation of Enroute Trajectory Change Requests
}

\author{
Husni Idris* \\ Engility Corporation, Billerica, MA 01821 \\ Stephanie Harrison, ${ }^{\dagger}$ and David Wing \\ NASA Langley Research Center, Hampton, VA 23681
}

\begin{abstract}
The advent of advanced technologies in communication, navigation, and surveillance is enabling more integration between the aircraft and the ground systems in managing air traffic operations. As a result, automation has evolved to provide the flight crew, air traffic controllers, and traffic flow managers with capabilities for collaborating on information access, analysis, and decision making. In this paper, we investigate different cooperative schemes between these agents, supported by automation, in managing dynamic trajectory changes while the flight is en route to improve flight and system performance. The analysis was conducted using an abstract cognitive tasking framework to identify trajectory change tasks independently from the agent performing them. Cooperation schemes were then derived by assessing different levels of cooperation on each task between the air and ground agents and their automation. The assessment was based on which automation-supported agent is more capable of performing the task and the expected benefit mechanisms that result from cooperating. The cooperation schemes were compared based on a qualitative, but objective, assessment of the benefits expected from cooperation.
\end{abstract}

\section{Introduction}

The advent of advanced technologies in communication, navigation, and surveillance is enabling more integration between the aircraft and the ground systems in managing air traffic operations. Aircraft equipped with the satellite-based automatic dependent surveillance-broadcast (ADS-B) can share awareness of their position and intent. Data communication (DataComm) enables more complex route changes, consisting of multiple waypoints, to be exchanged between the pilot and the air traffic controller than allowed by voice. Internet connectivity provides both the aircraft and the ground systems access to information such as wind and weather forecasts and traffic flow management restrictions. As a result, automation has evolved to provide the flight crew, air traffic controllers, and traffic flow managers with capabilities for collaborating on information access, analysis, and decision making.

NASA is undertaking a research effort to investigate different cooperation concepts between these agents in managing dynamic trajectory changes during en route flight to improve flight and system performance. In support of this research, this analysis was conducted to develop and compare concepts for integrating flight crew and airline dispatch activities regarding identifying and requesting an agreed upon route change from the service provider. The flight crew is supported by the Traffic Aware Planner (TAP), a cockpit automation system that monitors for route change opportunities that save time and/or fuel [1]. When found, routes are automatically probed and cleared of traffic and airspace hazards, known through ADS-B and internet connectivity. Then they are advised to the aircrew who requests the route change from the air traffic controller, using voice or DataComm if available. The tool makes aircrew requests better informed of airspace conditions and therefore more acceptable to the air traffic controller. The dispatcher is supported by the National Airspace System Constraint Evaluation and Notification Tool (NASCENT), an automation system that similarly monitors for route optimization opportunities and when found are advised to the dispatcher [2]. The route changes avoid weather hazards and are tested against flow management restrictions and airspace congestion. The dispatcher evaluates the recommended route based on fleet-wide objectives and constraints and, if desired, conveys the route to the aircrew so it may be requested from the controller. The

${ }^{*}$ Currently, Research Aerospace Engineer at NASA Ames Research Center, Flight Trajectory Dynamics \& Controls Branch, Mail Stop 210-10; husni.idris@nasa.gov. Senior Member, AIAA.

${ }^{\dagger}$ Aerospace Technologist, Aerospace Flight Systems; stephanie.harrison@nasa.gov. Senior Member, AIAA.

†Research Aerospace Technologist, Crew Station Systems; david.wing@nasa.gov. Senior Member, AIAA. 
dispatcher may also elect to submit the route change request to the appropriate traffic flow managers. This paper focuses on investigating approaches whereby the aircrew and the dispatcher, supported by the TAP and NASCENT automations, reconcile their route solutions before making a request from the service provider [3].

The analysis was conducted using an abstract cognitive tasking framework consisting of four phases: information acquisition, impact assessment, route selection, and route implementation. This framework helps identify tasks independently from the agent (air or ground, human or automation) who performs the task. Subtasks under each of the four cognitive phases were derived based on an operational scenario that involves identifying optimal route changes to mitigate an en route weather situation. Cooperation schemes were then derived by assessing different levels of cooperation on each task between the air and ground agents, based on which automation-supported agent is more capable of performing the task and the expected benefits that result from cooperating. The cooperation schemes were compared based on a qualitative, but objective, assessment of the benefits expected from cooperating on each task. The paper concludes with recommendations for the level of cooperation in each cognitive phase based on the capabilities and expected benefits.

\section{Approach}

The research approach consisted of five main activities, which are described in each of the following subsections:

\section{A. Identification of Key Concept Guidelines}

A number of guidelines were established to guide the development of the cooperative schemes. Namely, the agents and their automation tools should cooperate by leveraging their complementary capabilities and the company procedures should not be changed.

\section{B. Development of Operational Scenarios}

Operational scenarios were developed in order to provide a context for concept development. The scenario selected for this analysis was a dissipating weather system in which some flights remained on inefficient reroutes previously assigned to avoid the weather. The aircrew and dispatcher, using TAP and NASCENT respectively, would cooperate on selecting new reroutes that save time and/or fuel relative to the original reroute while avoiding constraints such as traffic and weather.

\section{Task Analysis}

A task analysis was performed in order to identify key tasks from the scenarios. An abstract cognitive tasking framework was used [4] to identify the tasks independently from the agents who may perform them. This abstraction is needed to identify cooperation schemes by allocating the abstracted tasks between the aircrew and dispatcher agents and between the human agent and the automation support. It consisted of four high level activities needed to identify and implement a route change request:

1) Information acquisition: Acquire information needed to identify a route change.

2) Impact assessment: Assess current and alternative routes against relevant performance metrics.

3) Route selection: Search for and identify improved routes.

4) Route implementation: Request the route change from the service provider.

Subtasks were identified under each of these activities and analyzed for potential cooperation between the agents in performing the task, as described in the next step.

\section{Cooperation Analysis}

Each task was analyzed for potential cooperation between the aircrew and dispatcher agents. This analysis was based on two factors: (1) which agent has a human and/or automation advantage to perform the task and cooperate on it, and (2) the benefit of different cooperation schemes on the task.

The ability to perform a task was defined as a combination of the three criteria [5]: the skill, rules, or knowledge to perform the task, the ability to access and process the information needed to perform the task, and the ability to produce the output of the task. The advantage of an agent to perform a task was distinguished as either inherent (i.e., only one of the agents has the capability to perform the task) or temporary (i.e., the development of comparable capability for the other agent can remove the advantage). For example, the aircraft's access to onboard weather radar 
information is an inherent advantage to the airborne agent as it cannot be produced on the ground. On the other hand, TAP currently generates altitude and lateral route changes while NASCENT generates only lateral route changes; however, a capability to add the altitude degree of freedom to NASCENT is possible and in fact planned. The analysis focused on inherent advantages, which included advantages that are impossible or difficult to remove given the current state of the technologies. The ability to cooperate on a task was defined as the ability to share information and produce a coordinated plan with the other agent [5].

A pilot, a dispatcher, and subject matter experts in the NASA automation tools (TAP and NASCENT) were consulted in determining the capabilities of each agent to perform and cooperate on each task. Then a desired scheme of cooperation on each task was postulated with the goal of increasing overall benefits from cooperation.

\section{E. Benefit Mechanism (BM) Assessment}

Different cooperation schemes were compared based on qualitative assessment of the expected benefits from cooperation. Benefit mechanisms from cooperating on each task were identified. Then they were abstracted into the following five generic benefit mechanisms, ranked qualitatively based on the relative magnitude of the quantitative benefits that may result from them.

Better Accuracy (BM1): Sharing the most accurate information and assessments between the agents results in reduced errors in the route solutions, which leads to greater satisfaction of user objectives and more accurate conformance to constraints. Fig. 1 shows a schematic of a route that has the potential to violate a hazard due to high uncertainty in the route and/or hazard. The reduction in uncertainty that results from sharing information and assessments leads to a reduction in the buffers that are needed to remain clear of such hazards. The reduced buffers in turn result in greater benefits (for example shorter routes, as shown in Fig. 1).

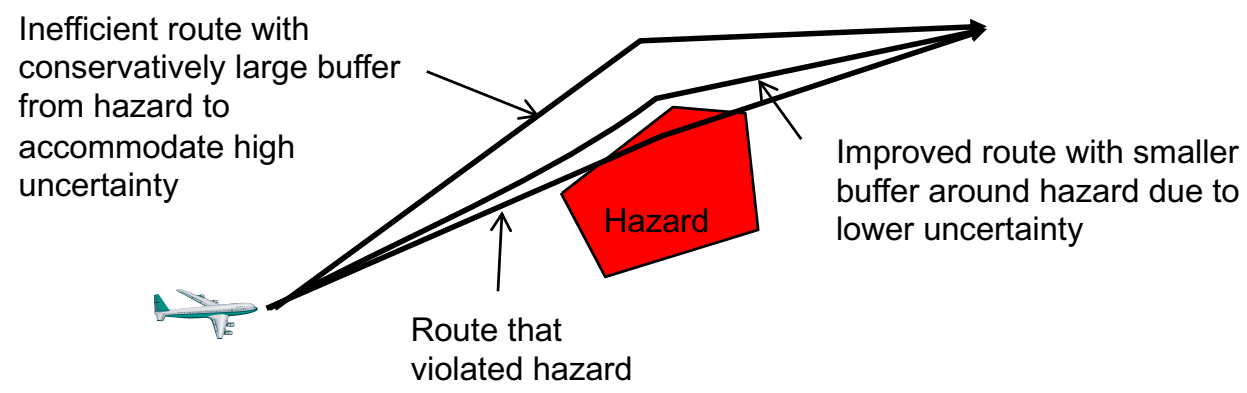

Fig. 1. Schematic of BM1 - Better Accuracy

Faster Response (BM2): Increased consistency by reconciling the air and ground information and assessments leads to faster convergence to solutions, which leads to faster approval of new routes, which finally leads to greater achievement of user objectives. Fig. 2 shows a schematic of BM2. A flight is on an inefficient route to the south of a hazard polygon. The flight has an opportunity to request a shorter route to the north of the hazard. As the flight continues to proceed along the inefficient route, the benefit opportunity diminishes, ultimately disappearing when the northern route becomes equal in length to the southern route. Faster communication and convergence, which may be achieved by consistency in information, assessments and solutions between the air and the ground, lead to faster approval by the service provider and a higher potential to achieve maximum benefit. Faster requests may also be enhanced by automated rather than manual communications, which may be accomplished through automated negotiation between TAP and NASCENT and using DataComm rather than voice in the request implementation.

BM2 leads to greater benefits than BM1 because BM2 leads to faster average service rate (more routes provided per unit time). On the other hand, BM1 leads to more accurate service (less probability of inefficient routes), reducing the variance of the service rate rather than increasing its average. 


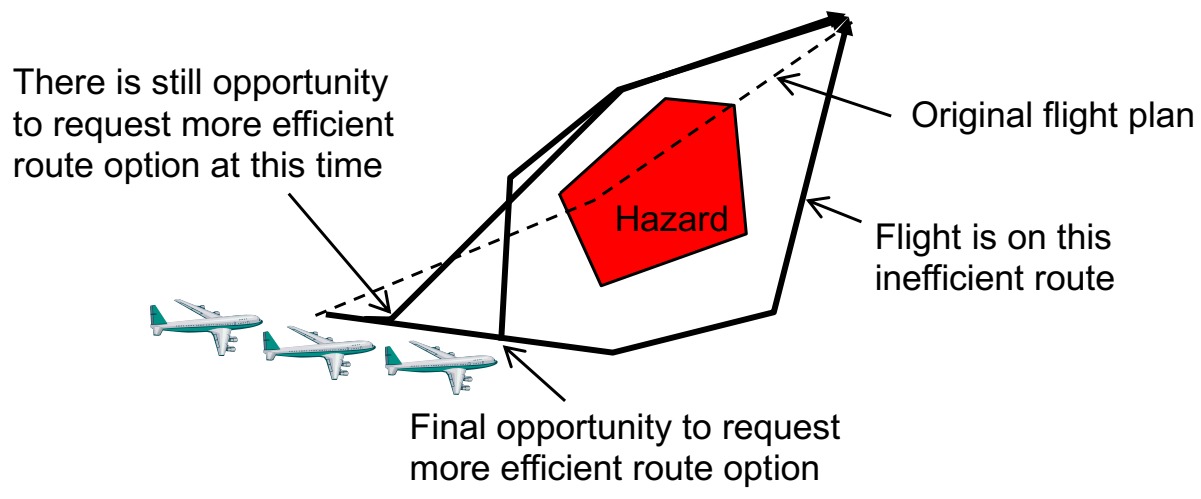

Fig. 2. Schematic of BM2 - Faster Response

System Optimality (BM3) is achieved, for example, by accounting for fleet-wide rather than single-flight objectives. Fig. 3 shows a schematic of BM3 where the dispatcher may decide to delay a particular flight while expediting another one competing for the same constrained resource (for example, a gap between weather cells or release from a holding pattern). The dispatcher justifies this decision by generating greater benefits (for example, time savings) for more flights at the expense of a few flights. The dispatcher is inherently more aware of the fleetwide impacts of a single flight solution than the flight crew.

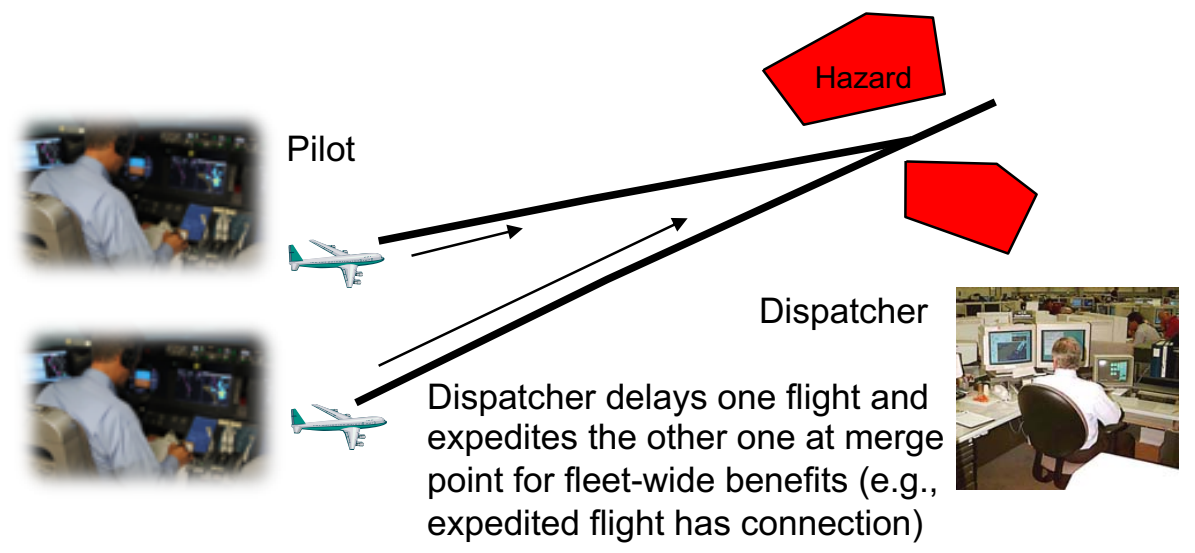

Fig. 3. Schematic of BM3 - System Optimality

BM3 has the potential to produce more benefits than BM2 because BM3 considers the benefits of several flights while BM1 and BM2 are single flight benefits. BM3 captures the multiplication of the benefits of BM1 and BM2 by several flights.

Higher Acceptability (BM4): Higher acceptability of requests to the service provider leads to greater achievement of user objectives. Fig. 4 shows a schematic of BM4 where a flight considers traffic congestion in deciding which route to request. One route is impacted by a congested sector while another one is not. Hence the second option has a higher chance of being accepted by air traffic service providers. Cooperating on information and assessments that increase awareness about the congestion (among other acceptability factors) leads to higher acceptability by the service provider. 


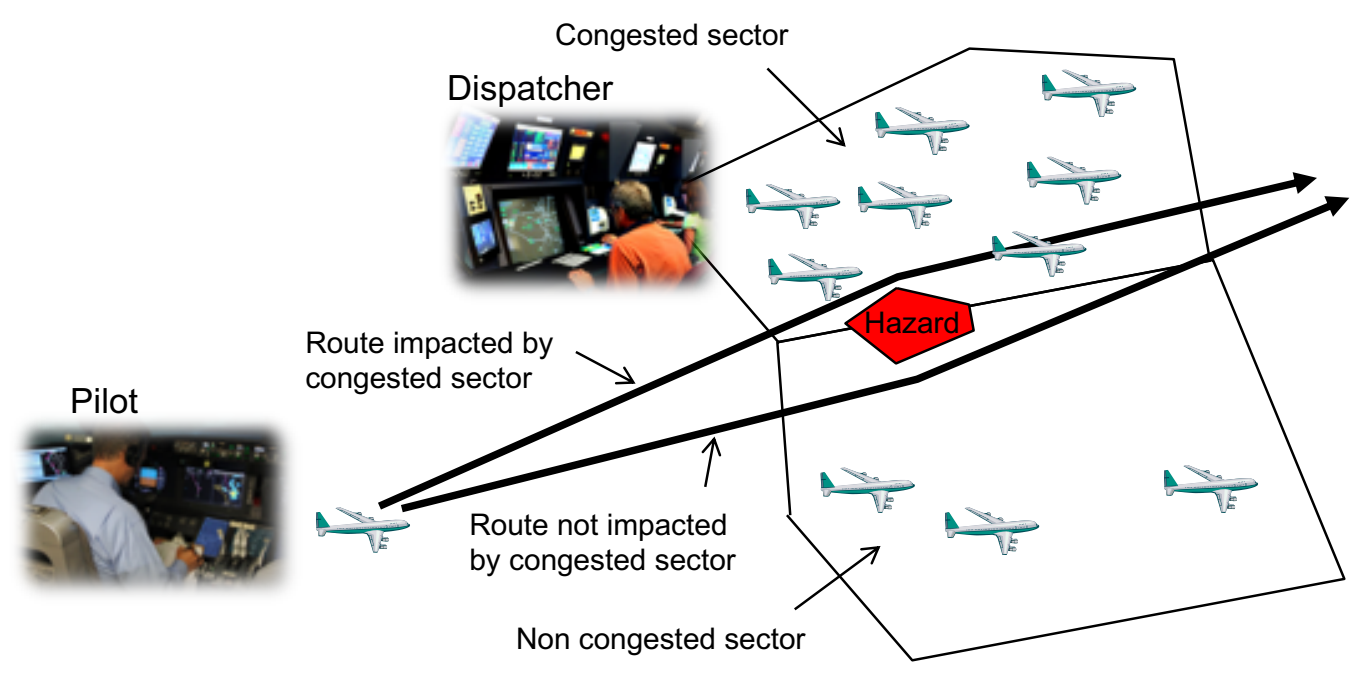

Fig. 4. Schematic of BM4 - Higher Acceptability

BM4 is ranked higher than BM3 because acceptability enables the intended benefits from all previous mechanisms.

More Flexibility (BM5): Cooperation can increase the overall route solution space by including the route solution subspaces considered by the cooperating agents. It enables more flexible routes to achieve greater user objectives. With the addition of automation support, such as using DataComm rather than voice communication, the agents can cooperated on more complex routes consisting of multiple waypoints. Fig. 5 shows a schematic of BM5, where a flight can request more route options that are also more complex, such as the route with multiple waypoints to fly through the gap between weather cells instead of the longer route around the weather.

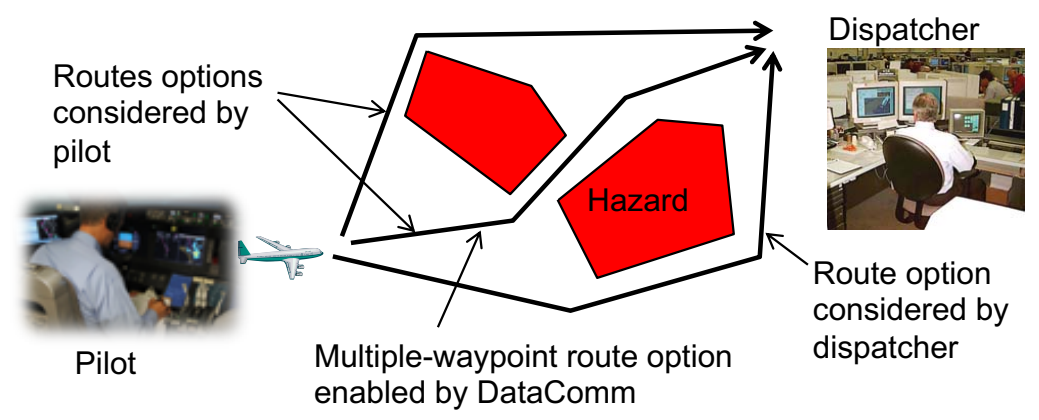

Fig. 5. Schematic of BM5 - More Flexibility

BM5 creates higher route capacity that can be used by multiple flights. In addition, BM5 reduces the air traffic controller's workload, which further increases capacity by allowing the controller to provide more route options to more flights. The increase in capacity is ranked higher than the benefits expected from the previous benefit mechanisms.

\section{Integrated Cooperation Analysis}

A list of forty subtasks under the four major tasks described in II.C were identified in the context of the recovery from weather scenario. Each subtask was analyzed as described in the approach and assigned desired cooperation schemes and corresponding benefit mechanisms. This detailed analysis is beyond the scope of the paper. In this section, we discuss the summary of the results for each of the four major cognitive tasks: information sharing, impact assessment, route selection, and route implementation. For each, we describe: (1) the cooperation between 
the air and ground agents based on the complementary nature of their inherent capabilities and (2) the role of the human and the automation in the cooperation. We discuss the corresponding benefit mechanisms that apply.

\section{A. Cooperation on information sharing}

Based on the detailed task analysis, some inherent differences between the air and ground agent's information were identified as shown in Table 1.

Table. 1. Advantages of Air versus Ground Information Capabilities

\begin{tabular}{|l|l|}
\hline \multicolumn{1}{|c|}{ Aircrew/TAP } & \multicolumn{1}{c|}{ Dispatch/NASCENT } \\
\hline $\begin{array}{l}\text { Access to onboard weather radar, out-the- } \\
\text { window weather, ADS-B traffic, and aircraft } \\
\text { weight/airspeed }\end{array}$ & $\begin{array}{l}\text { Access to fleet-wide objectives and constraints, } \\
\text { ground-based weather forecasts, and flow } \\
\text { restrictions }\end{array}$ \\
\hline
\end{tabular}

\section{Air-ground integration}

Cooperating on information sharing can be accomplished in part by accessing information from the same source, which can be done for static external information such as airspace structure and operating procedures and for dynamic external information such as flow restrictions. Cooperation on the inherent information differences highlighted in Table 1 requires exchanging the information between the agents. When the information is exchanged, it can be used by an agent to replace internal information that are inferior. For example, the aircraft weight and speed are more accurately known on the aircraft. They can be shared by the airborne agent (the pilot or the TAP automation) and the ground agent (dispatcher or NASCENT automation) can use it instead of their inaccurate estimates. The shared information can also be used to augment internal information with the shared information. Fig. 6 shows an example where the aircrew agent shares the onboard weather information (not known to the ground agent) and the dispatcher shares the ground-based weather information (not known to the aircrew) in an augmentative manner, leading them to a feasible route that avoids the weather systems known to either agent. Similarly, the dispatcher agent can share the fleet-wide objectives and constraints by instructing the aircrew, for example, to save as much time as possible, or save as much fuel as possible, or arrive at a specific waypoint no later than (NLT) a certain time to accommodate a schedule connectivity, or no earlier than (NET) a certain time to accommodate an unavailable gate. Such objectives and constraints stem from prioritizing among flights competing for routes and waypoints under congestion, about which the dispatcher is more aware than the aircrew.

\section{Feasible route by sharing weather information}

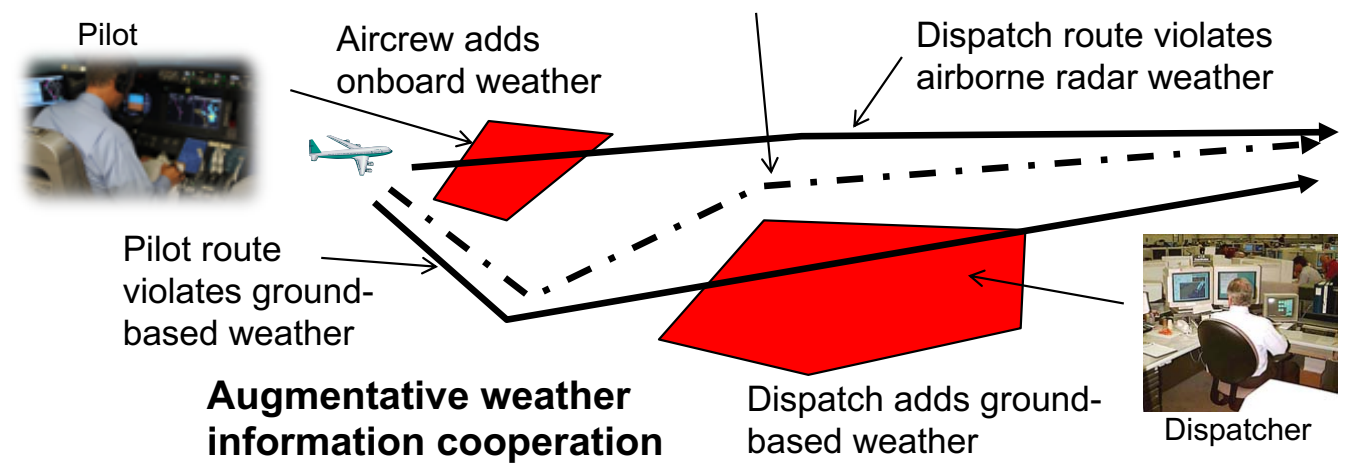

Fig. 6. Cooperation on Sharing Weather Information

Cooperation on information sharing leads to the following benefit mechanisms: (BM1) Sharing information would alleviate the discrepancy in the information and lead to more accurate solutions. For example, if the airborne agent knew about all the hazards known to the ground, it would select a route that is feasible with respect to all known hazards rather than the ones known to the airborne agent only, as shown in Fig. 6. (BM2) Cooperation on information sharing leads to making the route-change request faster, which in turn leads to a higher chance to seize 
the opportunity for improvement. For example, sharing the information would reduce the time needed to converge to a solution, because the air- and ground-generated routes at the start of cross checking would be more consistent. (BM3) In addition, sharing the fleet-wide objectives and constraints by the ground agent increases the benefits of the route change relative to single flight objectives.

\section{Role of automation}

Most information may be exchanged automatically between TAP and NASCENT without human involvement. In order to address communication bandwidth limitations, information that changes less dynamically, such as weather and traffic intent, can be transferred at lower rates than more rapidly changing information such as the aircraft weight and airspeed. Some information, such as the fleet-wide objectives and constraints, need to be entered by the dispatcher into NASCENT for transfer to TAP/aircrew, which avoids conveying such information to the aircrew by voice or legacy communication channels, reducing workload. Using appropriate algorithms, automation can assist in integrating exchanged information, such as weather, more accurately and faster than the human operators are able to, enhancing benefits from BM1 and BM2, respectively.

\section{B. Cooperation on impact assessment}

Based on the detailed task analysis, Table 2 lists assessment capabilities in which one agent has an advantage due to availability of information or fidelity of trajectory modeling.

Table. 2. Advantages of Air versus Ground Impact Assessment Capabilities

\begin{tabular}{|l|l|}
\hline \multicolumn{1}{|c|}{ Aircrew/TAP } & \multicolumn{1}{c|}{ Dispatch/NASCENT } \\
\hline (1) Assess impact of nearby weather & (1) Assess impact on traffic congestion and \\
detected using onboard radar or pilot's out- & flow constraints. (2) Assess impact on fleet- \\
the-window view. (2) Assess proximity to & wide considerations such as downstream \\
traffic due to high fidelity trajectory & schedules due to dispatcher knowledge of \\
modeling and access to ADS-B traffic. & fleet-wide concerns. \\
\hline
\end{tabular}

\section{Air-ground integration}

Cooperation on impact assessment in addition to information sharing alleviates the adverse effects of the differences in information between the air and the ground agents, which are due to either inherent or technical limitations. An agent sends a route candidate to the other agent for assessment against its analysis capabilities. In the scenario of Fig. 6, NASCENT queries TAP about the impacts of its route relative to airborne weather and ADS-B traffic hazards known by TAP. TAP queries NASCENT about the impacts of its route candidate on traffic congestion and fleet-wide concerns. After sufficient iterations, the agents would converge to a feasible route that accommodates all known constraints and objectives.

The cooperation on impact assessment results in the following benefit mechanisms: (BM1) Sharing impacts can alleviate the discrepancies in information that cannot be shared explicitly or analyzed by either agent. This increases the accuracy of a route change. (BM2) Sharing impacts reduces the time needed to reconcile a route change by reducing the time needed to negotiate between the agents. (BM3) Sharing the impact of a route on fleet-wide considerations by the ground agent increases the achievement of fleet-wide benefits. (BM4) Sharing impacts on traffic proximity by TAP and on traffic congestion and flow restrictions by NASCENT increases the acceptability of the route change by the service provider.

\section{Role of automation}

Automated exchange of routes and impact assessments between the automation tools (TAP and NASCENT) would speed up the convergence to the desired route solution (BM2). However, there are a number of concerns with the automation of one agent invoking the automation of the other agent without a human input: (1) Airline procedures require concurrence between the pilot and dispatcher on route changes that exceed predefined criteria. Hence, the automation may query the other automation for changes within the criteria while larger changes may have to be reserved for invocation only by the human operator to avoid confusing human versus automation concurrence. Additionally, guards may be placed such that no human concurrence is requested by the automation unless the other human operator initiated the request. (2) Automated queries may result in congestion of the communication line versus human invoked queries, which only occur when desired by the human. 


\section{Cooperation on route selection}

Based on the detailed task analysis, Table 3 lists some of the parameters used differently by the route selection of the air and the ground agents.

Table. 3. Advantages of Air versus Ground Route Selection Capabilities

\begin{tabular}{|l|l|}
\hline \multicolumn{1}{|c|}{ Aircrew/TAP } & \multicolumn{1}{c|}{ Dispatch/NASCENT } \\
\hline Immediate reroute start point & Delayed reroute start point \\
Pilot selected return point & Historically used return point \\
Lateral and vertical degrees of freedom & Lateral degrees of freedom (vertical is planned) \\
\hline
\end{tabular}

\section{Air-ground integration}

Even if cooperation was performed on information sharing and impact assessment, inefficiencies may still arise because each agent adopts a different set of assumptions and parameters in selecting its route. For example, as shown in Fig. 7, NASCENT typically uses a starting point farther away from the current aircraft position, relative to TAP, to account for additional communication and coordination needs. NASCENT also uses a return waypoint (to the original route) that reconciles historically acceptable waypoints by air traffic control while TAP's return point is entered by the aircrew. Cooperation on route selection can alleviate some of the adverse effects of these inconsistencies and/or leverage their complementary benefits.

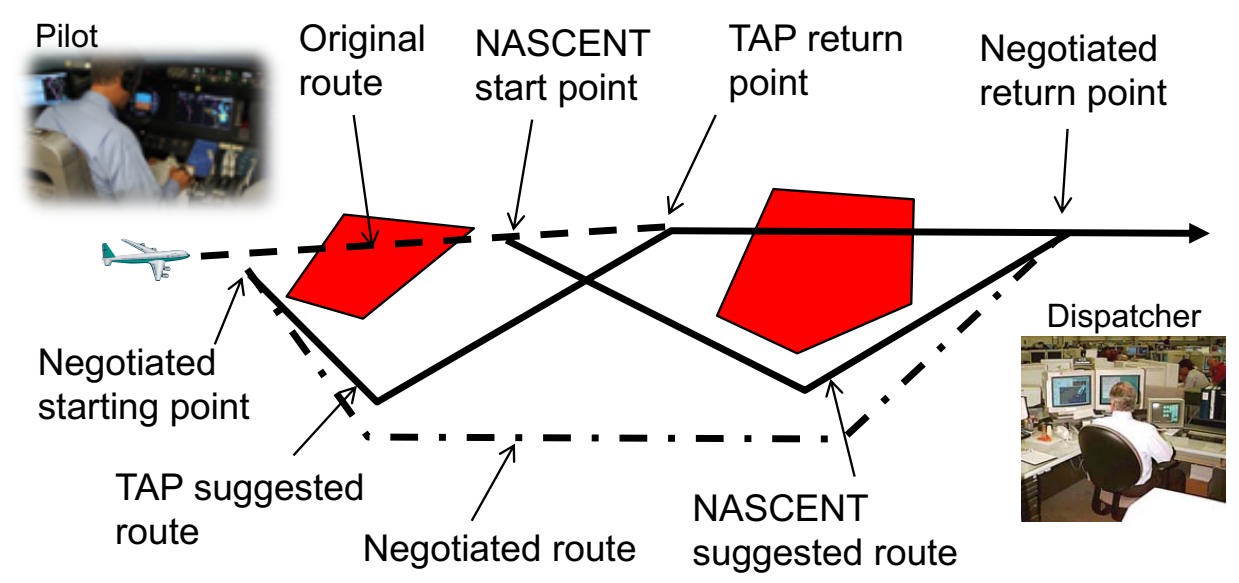

Fig. 7. Cooperation on Route Selection

If no concurrence between the pilot and the dispatcher is required by airline procedures, the flight crew can make a choice of the better route change suggested by TAP and NASCENT. The judgment may include objective and subjective concerns of the aircrew. If concurrence is needed a negotiation between the human agents is ultimately required. An augmentative or integrative cooperation scheme can also be used to generate a single route by reconciling route elements considered by each agent. There are several aspects of the route that can be cooperated on, including the starting point, the return point, the degrees of freedom, and the set of additional waypoints considered, as listed in Table 3. These parameters can be shared and then used as additional constraints or objectives to generate a reconciled route change that is agreeable to both agents. For example, in Fig. 7, a negotiated route was ultimately selected using the starting point advised by TAP, taking advantage of faster communication, and the return point advised by NASCENT, taking advantage of historically acceptable routes.

Cooperation on route selection can result in the following benefit mechanisms: (BM3) Using constraints such as NET or NLT at certain waypoints may be used by the dispatcher to increase the fleet-wide objectives. (BM4) Reconciling return points that are more acceptable by the service provider (such as historically defined return points) increases the acceptability of the route by the service provider. (BM5) Augmenting the degrees of freedom and the additional waypoints considered by each agent increases the size and flexibility of the solution space. 


\section{Role of automation}

The route selection cooperation can be automated where TAP and NASCENT engage in a negotiation (iteration on solutions and cross checking their impacts) until they converge to a route that they would present to the human operators. For small route changes, the route may be presented only to the pilot if dispatcher concurrence is not required by the airline policy. The human operators may only be involved to interrupt an automated negotiation that takes too long or to initiate an alternative manual route selection process when desired. The benefit of automated negotiation is speeding up the route selection process (BM2) and in some cases, increasing the fleet-wide optimality (BM3) and flexibility (BM4) of the solution by considering more solutions and complex objectives than the human operators are able to consider. However, automated negotiation requires a more advanced state of automation sophistication and is envisioned as a more futuristic cooperation scheme.

\section{Cooperation on route implementation}

Based on the detailed task analysis, Table 4 lists the difference in route implementation between the air and the ground agents.

Table. 4. Advantages of Air versus Ground Route Implementation Capabilities

\begin{tabular}{|l|l|}
\hline \multicolumn{1}{|c|}{ Aircrew/TAP } & \multicolumn{1}{c|}{ Dispatch/NASCENT } \\
\hline $\begin{array}{l}\text { Voice or DataComm route change request } \\
\text { from controller }\end{array}$ & $\begin{array}{l}\text { Send route change to aircrew or request it from } \\
\text { traffic flow manager by voice or using collaborative } \\
\text { trajectory options program (CTOP) }\end{array}$ \\
\hline
\end{tabular}

\section{Air-ground integration}

Cooperation on making the route change request can offer the following additional benefits:

After negotiating the route change between the airborne and ground agents, the flight crew makes the route change request from the air traffic controller. The flight crew is more suited to making route change requests that impact the current air traffic controller. For example, the pilot can assess, based on the workload of the air traffic controller, when to make the request, particularly if made by voice. The dispatcher can cooperate by making strategic route requests that impact the route further downstream from the current controller or the current facility. This helps increase the potential that the route change is accepted by avoiding the workload limitation of the current controller (BM4). In addition, the request can be submitted digitally, for example through the collaborative trajectory options program (CTOP), instead of using voice.

\section{Role of automation}

If DataComm is used for the route change request instead of voice, a larger number of waypoints can be included in the route change, which allows more route solutions to avoid complex weather systems and other constraints (BM5). While this is not explicitly a cooperation benefit, the automation can build on it by using the added flexibility in determining and negotiating more optimal routes. With digital communication, it is possible to deliver the route change requests to the service provider with different levels of human involvement: (1) the human operator always initiates the request delivery, (2) the human operator vetoes the automated delivery, and (3) the human is ignored while the automation delivers the request. The second and third levels of automation are extreme and not envisioned to be feasible in the near future as they require high automation sophistication and operator trust in the automation. The first level is envisioned possible and desired in the near future, resulting in reduced workload of the human operators. It should be noted that even if the automation bypasses the pilot in making requests, the air traffic controller is always able to evaluate the safety of a request and reject or accept it accordingly.

\section{Conclusions}

In summary, cooperation schemes between the aircrew and dispatchers, and their automation tools, to agree on route change requests made to the service provider were identified and analyzed. Function allocation and benefit mechanism analyses were combined to identify key cooperation areas with high potential benefits. The analysis resulted in generic characterization of the benefit mechanisms that are enabled by different levels of cooperation between the air and the ground agents, and how their automation support affects and enhances these benefit mechanisms. Table 5 shows a qualitative representation of this characterization, highlighting the locus of benefit mechanisms achieved by cooperating on each major cognitive task. The highest benefits are expected from the 
following cooperation areas: (1) Cooperation on sharing information such as weather, traffic, aircraft performance parameters, and fleet-wide objectives increases the accuracy of the route changes, reduces the time needed for reconciliation and concurrence, and increases the system-wide optimality of the route change. (2) Cooperating by cross-checking route impacts increases the acceptability of the route change requests by the service provider, in addition to furthering the benefits of making the route change more accurate, responsive, and globally optimal. The cooperation leverages the airborne automation capabilities of avoiding ADS-B traffic and onboard radar weather hazards and the ground automation capabilities of assessing the impacts on traffic congestion, ground-based weather hazards and flow restrictions, (3) Cooperating on selecting the route change further increases the benefits of global optimality and acceptability, through reconciling fleet-wide objectives and constraints and reconciling route parameters, such as start and return waypoints that are more acceptable. It also increases the flexibility of the route change by considering additional degrees of freedom and waypoints considered by either the airborne and ground agents. (4) Cooperating on implementing the route change requests using digital means rather than voice, such as DataComm by the aircrew and CTOP by the dispatcher, increases route acceptability and flexibility by enabling complex multi-waypoint routes and avoiding increasing controller workload.

Table. 5. Benefit Mechanisms Characterization of Cooperation Levels

\begin{tabular}{|l|c|c|c|c|c|}
\hline & $\begin{array}{c}\text { BM1: More } \\
\text { Accuracy }\end{array}$ & $\begin{array}{c}\text { BM2: Faster } \\
\text { Response }\end{array}$ & $\begin{array}{c}\text { BM3: System } \\
\text { Optimality }\end{array}$ & $\begin{array}{c}\text { BM4: } \\
\text { Higher } \\
\text { Acceptability }\end{array}$ & $\begin{array}{c}\text { BM5: More } \\
\text { Flexibility }\end{array}$ \\
\hline $\begin{array}{l}\text { Information } \\
\text { Sharing }\end{array}$ & $\mathrm{X}$ & $\mathrm{X}$ & $\mathrm{X}$ & & \\
\hline $\begin{array}{l}\text { Impact } \\
\text { Assessment }\end{array}$ & $\mathrm{X}$ & $\mathrm{X}$ & $\mathrm{X}$ & $\mathrm{X}$ & \\
\hline Route Selection & & & $\mathrm{X}$ & $\mathrm{X}$ & $\mathrm{X}$ \\
\hline $\begin{array}{l}\text { Route } \\
\text { Implementation }\end{array}$ & & & & $\mathrm{X}$ & $\mathrm{X}$ \\
\hline
\end{tabular}

This characterization can be used qualitatively to assess whether the cooperation should be in sharing the information only, extend to cooperating on impact assessments, extend to cooperating on the solution selection, or extend all the way to cooperating on the implementation, based on benefit mechanisms. The benefit mechanisms can then serve as a first step towards more objective and quantitative benefit assessment and comparison between cooperation schemes.

\section{References}

[1] D. Wing, M. Ballin, S. Koczo, R. Vivona, and J. Henderson, "Developing an Onboard Traffic-Aware Flight Optimization Capability for Near-Term Low-Cost Implementation," 13th AIAA Aviation Technology, Integration, and Operations Conference, Los Angeles, CA, August 2013.

[2] K., Sheth, D. McNally, P. Somersall, A. Morando, A. Clymer, and F. Shih, "Assessment of a National Airspace System Airborne Rerouting Tool," 11th USA/Europe Air Traffic Management Research and Development Seminar, Lisbon, Portugal, 2015

[3] NASA TM. Airspace Technology Demonstration-3 (ATD-3) En Route Concept of Operations Synopsis Version 0.9, 2015.

[4] H. Idris, G. Enea, and T. Lewis, Idris, H., Enea G., and Lewis T., "Function Allocation between Automation and Human Pilot for Airborne Separation Assurance," IFAC/IFIP/IFORS/IEA Symposium on Analysis, Design, and Evaluation of Human-Machine Systems, Kyoto, Japan, 2016.

[5] P. Millot and M-P. Pacaux-Lemoine, "A Common Work Space for a mutual enrichment of Human-machine Cooperation and Team-Situation Awareness," 12th IFAC Symposium on Analysis, Design, and Evaluation of Human-Machine Systems August 11-15, 2013. Las Vegas, NV, USA. 